\title{
Spin-Induced Scalarized Black Holes
}

\author{
Carlos A. R. Herdeiro $\odot,{ }^{1}$ Eugen Radu, ${ }^{1}$ Hector O. Silva $\odot,{ }^{2,3}$ Thomas P. Sotiriou, ${ }^{4}$ and Nicolás Yunes $\odot^{3}$ \\ ${ }^{1}$ Departamento de Matemática da Universidade de Aveiro and CIDMA, Campus de Santiago, 3810-183 Aveiro, Portugal \\ ${ }^{2}$ Max Planck Institute for Gravitational Physics (Albert Einstein Institute), Am Mühlenberg 1, Potsdam 14476, Germany \\ ${ }^{3}$ Illinois Center for Advanced Studies of the Universe and Department of Physics, University of Illinois at Urbana-Champaign, \\ Urbana, Illinois 61801, USA \\ ${ }^{4}$ School of Mathematical Sciences and School of Physics and Astronomy, University of Nottingham, \\ University Park, Nottingham, NG7 2RD, United Kingdom
}

(Received 8 September 2020; accepted 20 November 2020; published 7 January 2021)

\begin{abstract}
It was recently shown that a scalar field suitably coupled to the Gauss-Bonnet invariant $\mathcal{G}$ can undergo a spin-induced linear tachyonic instability near a Kerr black hole. This instability appears only once the dimensionless spin $j$ is sufficiently large, that is, $j \gtrsim 0.5$. A tachyonic instability is the hallmark of spontaneous scalarization. Focusing, for illustrative purposes, on a class of theories that do exhibit this instability, we show that stationary, rotating black hole solutions do indeed have scalar hair once the spininduced instability threshold is exceeded, while black holes that lie below the threshold are described by the Kerr solution. Our results provide strong support for spin-induced black hole scalarization.
\end{abstract}

DOI: 10.1103/PhysRevLett.126.011103

Introduction.-Black holes (BHs) are central players in astrophysics. The recent detections of gravitational waves $[1,2]$ and the first $\mathrm{BH}$ imaging [3] have consolidated the evidence for their physical reality. Under the leading paradigm, astrophysical BHs are described by the Kerr metric [4]. Astonishingly, this hypothesis entails this macroscopic class of objects, ranging 10 orders of magnitude in mass, having only 2 (macroscopic) degrees of freedom: mass $M$ and spin $J$.

A tantalizing possibility beyond the Kerr hypothesis is that astrophysical BHs are not described by the Kerr metric only in certain regimes. For instance, if ultralight bosonic fields exist, e.g., as dark matter, they may undergo a superradiant instability near Kerr BHs [5], forming a bosonic cloud [6], which, in some cases, leads to new stationary BHs [7-9]. The instability, however, is only efficient for a range of $\mathrm{BH}$ masses determined by the ultralight field's mass [10-12].

The prospect of such elusive non-Kerr BHs takes a different guise in gravity theories that allow BH scalarization $[13,14]$. Theories that fashion a coupling between a scalar and the Gauss-Bonnet invariant can exhibit a tachyonic instability near BHs when the BH spin exceeds a certain threshold [15]. Interestingly, crossing that threshold also allows these models to circumvent a known no-hair theorem $[13,15]$. Hence, one expects that stationary BHs in these models will exhibit spin-induced scalar hair only when they are rapidly spinning. As we show below, this is indeed the case.

Spontaneous scalarization.-This effect was first discussed by Damour and Esposito-Farèse (DEF) [16,17] for compact stars in scalar-tensor theories of gravity. The
DEF model demonstrated that, if suitably coupled to gravity, a new field could go undetected in weak field tests of general relativity (GR) and still have an influence in the strong field of neutron stars, providing strong motivation for GR tests with binary pulsars. Indeed, the latter have severely constrained the DEF model [18-20], although the constraints can be evaded if the field is massive [21].

In the DEF model (massless or massive), scalarization happens only for stars and does not affect BHs [22] since, in fact, the model is covered by no-hair theorems [25-27]. However, it was recently shown that scalar-tensor theories that exhibit BH scalarization do exist $[13,14]$. Consider a scalar-Gauss-Bonnet (sGB) theory with action

$$
S=\frac{1}{16 \pi} \int d^{4} x \sqrt{-g}\left[R-2 \partial_{\mu} \phi \partial^{\mu} \phi+\lambda^{2} f(\phi) \mathcal{G}\right],
$$

where $\mathcal{G} \equiv R^{\mu \nu \rho \sigma} R_{\mu \nu \rho \sigma}-4 R^{\mu \nu} R_{\mu \nu}+R^{2}$ is the GaussBonnet invariant, $\lambda$ (with units of length) determines the coupling strength between scalar field and $\mathcal{G}$, and $f$ is a dimensionless function of the scalar field $\phi$. (We work with units where $G=1=c$ ). If $f^{\prime}\left(\phi_{0}\right)=0$ [28], for some constant $\phi_{0}$, GR vacuum solutions, together with $\phi=\phi_{0}=$ constant, are admissible solutions of the field equations derived from Eq. (1). This condition excludes the dilatonic $[f \propto \exp (\phi)]$ and shift-symmetric $(f \propto \phi)$ subclasses of sGB in which BHs always have scalar hair [27,29-31]. The constant $\phi_{0}$ solutions are, in fact, unique thanks to a no-hair theorem [13], provided that

$$
f^{\prime \prime}\left(\phi_{0}\right) \mathcal{G}<0
$$


Interestingly, $-\lambda^{2} f^{\prime \prime}\left(\phi_{0}\right) \mathcal{G} / 4$ is the effective mass squared for scalar field perturbations around the GR solution, and, in this sense, the condition in Eq. (2) ensures the absence of tachyonic instabilities.

This suggests that scalarization can occur if Eq. (2) is violated. Indeed, as a simple example consider the choice $f(\phi)=\phi^{2} / 2$. For $\phi=0$, the Schwarzschild $\mathrm{BH}$ is an admissible solution and $\mathcal{G}=48 M^{2} / r^{6}$, where $M$ is the Arnowitt-Deser-Misner (ADM) mass. Evaluated on the horizon, the effective mass squared of scalar perturbations is then $-3 \lambda^{2} /\left(16 M^{4}\right)$, indicating the possibility that a tachyonic instability can take place. In general, the effective mass can be somewhat negative and still have a stable configuration [32], but the scalar field perturbation will become unstable if the dimensionless ratio $M / \lambda$ is made sufficiently small. In practice, if $M / \lambda \lesssim 0.587$, the scalar field will develop a tachyonic instability, whose end point might be a scalarized $\mathrm{BH}$ [13].

The fact that the onset of scalarization is captured in linear theory allows one to identify all possible couplings to curvature that can lead to scalarization [34] and simplifies the investigation of the relevant thresholds [35]. It also makes it straightforward to generalize the mechanism to nongravitational couplings (see, e.g., [36,37]). However, the end point of the instability depends on nonlinear interactions, as these are the ones that eventually quench the linear instability. For example, although static, spherically symmetric scalarized BHs exist for both $f(\phi) \propto \phi^{2}$ [13] and $f(\phi) \propto e^{\phi^{2}}$ [14], they have different radial stability properties [38]. This can be attributed to the additional nonlinear interactions between $\phi$ and $\mathcal{G}$ in the second model [39]. Alternatively, supplementing the simplest choice, $f(\phi)=\phi^{2} / 2$, which already determines fully the onset of scalarization, with a nonlinear potential for the scalar also yields radially stable (and entropically preferred) scalarized BHs [40].

BH rotation.-The effect of rotation on $\mathrm{BH}$ scalarization was considered in Ref. [41] for the choice

$$
f(\phi)=\frac{\epsilon}{12}\left(1-e^{-6 \phi^{2}}\right),
$$

and $\epsilon=+1$ [42]. It was shown that rotation tends to suppress scalarization. This can be partially understood in an intuitive manner as follows. For a Kerr BH in BoyerLindquist coordinates $(t, r, \theta, \varphi)$, one has

$$
\mathcal{G}_{\text {Kerr }}=\frac{48 M^{2}}{\left(r^{2}+\chi^{2}\right)^{6}}\left(r^{6}-15 r^{4} \chi^{2}+15 r^{2} \chi^{4}-\chi^{6}\right),
$$

where $\chi \equiv a \cos \theta, a=J / M$ is the Kerr spin (per unit mass) parameter, where $J$ is the angular momentum. When $a=0$, one recovers the Schwarzschild metric, where $\mathcal{G}$ is positive definite and monotonic in $r$. For the Kerr metric, as long as the dimensionless spin $j \equiv a / M \leq 0.5, \mathcal{G}$ remains positive definite and the spacetime is said to be "gravitoelectric dominated." However, this is no longer true when $j>0.5$ and regions of "gravitomagnetic dominance" in which $\mathcal{G}$ is negative arise for some neighborhoods of $r, \theta$ [43]. Thus, rotation can make the effective mass of the scalar field less negative or even positive near the horizon for $\epsilon=+1$ and therefore suppress the effect of scalarization.

The focus on $\epsilon=+1$ is motivated by the fact that, in the absence of rotation, it is a necessary condition for $\mathrm{BH}$ scalarization. However, the last observation about $\mathcal{G}_{\text {Kerr }}$ suggests that $\mathrm{BH}$ spin might be able to induce scalarization when $\epsilon=-1$. Indeed, it was shown recently in Ref. [15] (see also [44,45] for follow-up studies) that Kerr BHs are tachyonically unstable for $f(\phi)=\epsilon \phi^{2} / 2$ and $\epsilon=-1$ once $j$ exceeds a certain threshold (which is above $j=0.5$ ). Since this tachyonic instability is the hallmark of spontaneous scalarization, one expects theories in this class to exhibit a remarkable property: BHs develop scalar hair only when they spin fast enough.

The approach of Ref. [15], however, does not provide concrete evidence that these hairy BHs exist. As it focuses on the linearized equations, it captures only the onset of the tachyonic instability, and it cannot make conclusive statements about its end point. In this Letter, we instead solve the full field equations numerically to generate stationary, rotating, asymptotically flat $\mathrm{BH}$ solutions. We show that slowly rotating $\mathrm{BHs}$ can only be described by the Kerr solution, as in GR, whereas, rapidly rotating ones, can indeed have scalar hair. This is fully consistent with the expectations of Ref. [15] and a clear demonstration that rotation can induce scalar hair if a scalar field exhibits suitable coupling to curvature.

Nonlinear spin-induced scalarized BHs. - We work with the coupling of Eq. (3) and $\epsilon=-1$. At the linear level, this theory coincides with the model studied in [15], but the end state of the instability, which is our focus, is sensitive to the nonlinear completion of the theory. We use the exponential model mostly to facilitate a comparison between our results and those of Ref. [41], which studied the case $\epsilon=+1$. We stress that other couplings $f(\phi)$ could have been chosen, including the quadratic model $f(\phi)=\epsilon \phi^{2} / 2$ or the effective-field-theory-inspired model of [40]. We expect all these models to also exhibit the spin-induced spontaneous BHs' scalarization effect, although the nonlinear solutions will have different properties [38-40].

To find these solutions, we work with the ansatz [41]

$$
\begin{aligned}
d s^{2}= & -e^{2 F_{0}} N d t^{2}+e^{2 F_{1}}\left(N^{-1} d r^{2}+r^{2} \mathrm{~d} \theta^{2}\right) \\
& +e^{2 F_{2}} r^{2} \sin ^{2} \theta(d \varphi-W d t)^{2},
\end{aligned}
$$

where $N \equiv 1-r_{H} / r$ and $r=r_{H}>0$ is the horizon location [46]. The metric functions $F_{i}, W(i=0,1,2)$ and the scalar field $\phi$ depend on $r, \theta$ only. Asymptotic flatness requires $\lim _{r \rightarrow \infty} F_{i}=\lim _{r \rightarrow \infty} W=\lim _{r \rightarrow \infty} \phi=0$. Axial symmetry and regularity impose the boundary conditions 
$\partial_{\theta} F_{i}=\partial_{\theta} W=\partial_{\theta} \phi=0$ on the symmetry axis $(\theta=0, \pi)$. Additionally, the absence of conical singularities implies that $F_{1}=F_{2}$ on the symmetry axis. The horizon boundary conditions are $\left.\partial_{x} F_{i}\right|_{r=r_{H}}=\left.\partial_{x} \phi\right|_{r=r_{H}}=0$ and $\left.W\right|_{r=r_{H}}=\Omega_{H}$, where, for convenience, we have introduced a new radial coordinate $x \equiv\left(r^{2}-r_{H}^{2}\right)^{1 / 2}$. Here $\Omega_{H}>0$ is the constant horizon angular velocity. Some details on the numerical scheme used to find the solutions with these boundary conditions are given in the Supplemental Material, Sec. II [47].

Most of the quantities of interest are encapsulated in the metric functions evaluated either at the horizon or at infinity. Consider first horizon quantities. The Hawking temperature is $T_{H}=\kappa /(2 \pi)$, where $\kappa$ is the surface gravity defined as $\kappa^{2} \equiv-\left.(1 / 2)\left(\nabla_{\alpha} \xi_{\beta}\right)\left(\nabla^{\alpha} \xi^{\beta}\right)\right|_{r_{H}}$ and $\xi \equiv \partial_{t}+$ $\Omega_{H} \partial_{\varphi}$ is the horizon null generator. The area of the spatial sections of the event horizon is $A_{H}$. Explicitly,

$$
\begin{gathered}
T_{H}=\left(4 \pi r_{H}\right)^{-1} \cdot e^{F_{0}\left(r_{H}, \theta\right)-F_{1}\left(r_{H}, \theta\right)}, \\
A_{H}=2 \pi r_{H}^{2} \int_{0}^{\pi} d \theta \sin \theta e^{F_{1}\left(r_{H}, \theta\right)+F_{2}\left(r_{H}, \theta\right)} .
\end{gathered}
$$

Now consider the asymptotic quantities. The ADM mass $M$ and the angular momentum $J$ are read off from the asymptotic behavior of the metric functions: $g_{\mathrm{tt}} \simeq-1+$ $2 M / r$ and $g_{\varphi t} \simeq-2 J \sin ^{2} \theta / r$. All solutions reported in this Letter possess also a scalar "charge" $Q_{s}$, which is found from the scalar field's far-field asymptotic $\phi \simeq-Q_{s} / r$. This "charge" does not have an associated conservation law, and it is secondary in the nomenclature of Refs. [29,49,50]. For all solutions here, both the metric functions and the scalar field are even parity, i.e., invariant with respect to the transformation $\theta \rightarrow \pi-\theta$. More general solutions, in particular with odd parity, exist. Typically these are excited states and unstable, which justifies our focus on the even parity sector, corresponding to the fundamental solutions [51].

As in the $\epsilon=+1$ [41] case, the solutions satisfy a Smarrtype law, and their entropy $S$ has a correction to the Bekenstein-Hawking entropy computed from Wald's formalism [52]. It reads $S=S_{E}+S_{\mathrm{SGB}}$, where

$$
S_{E}=\frac{A_{H}}{4}, \quad S_{\mathrm{sGB}}=\frac{\lambda^{2}}{2} \int_{H} d^{2} x \sqrt{h} f(\phi) R^{(2)},
$$

with $R^{(2)}$ denoting the Ricci scalar of the metric $h_{i j}$, which is induced on the spatial sections of the horizon, denoted as $H$. In the following, we shall use the dimensionless (or reduced) area $a_{H} \equiv A_{H} /\left(16 \pi M^{2}\right)$, spin $j=J / M^{2}$, temperature $t_{H} \equiv 8 \pi T_{H} M$, and entropy $s \equiv S /\left(4 \pi M^{2}\right)$.

Properties of the solutions. - We have performed a thorough numerical exploration of the parameter space to examine the domain of existence and the physical properties of the spinning scalarized BHs. This domain
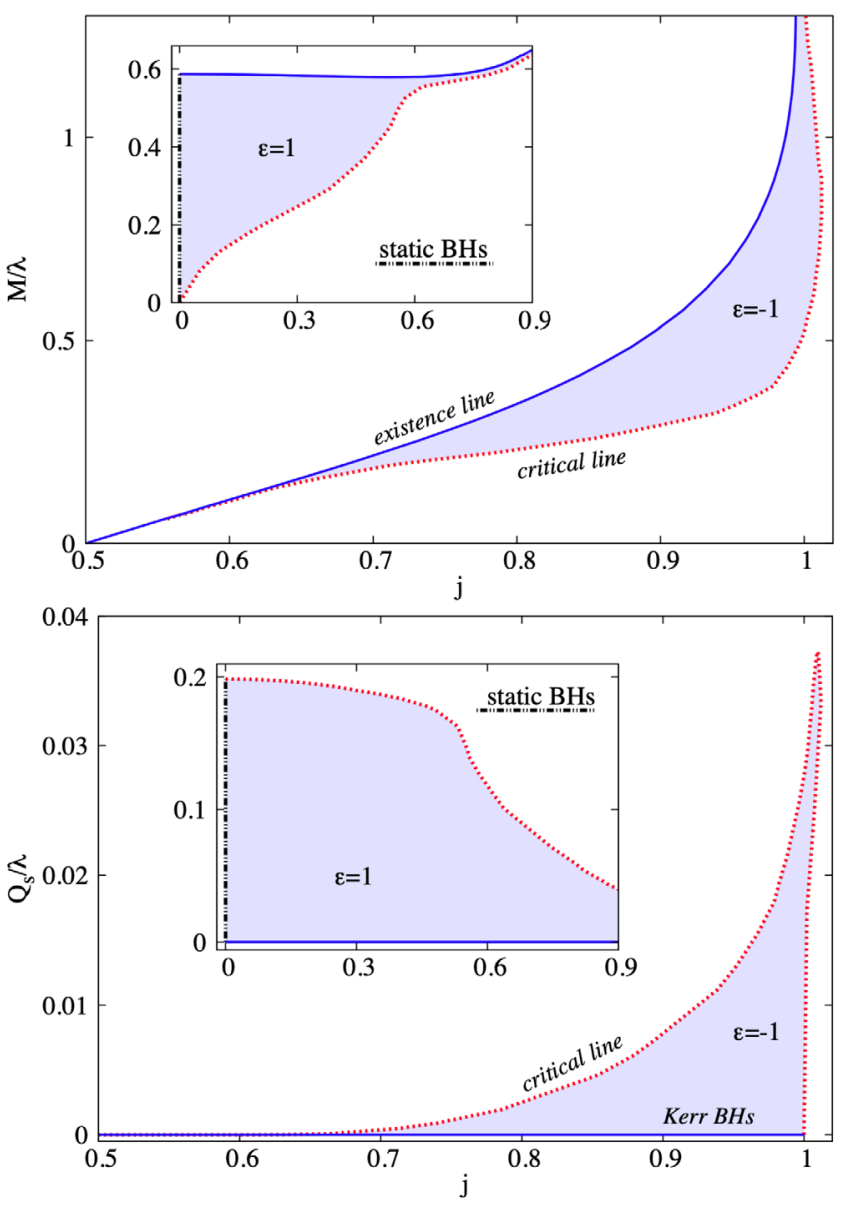

FIG. 1. ADM mass $M$ (top panel) and scalar charge $Q_{s}$ (bottom panel), both in units of $\lambda$, as functions of the dimensionless spin $j$ of spinning scalarized BHs. Here and in Fig. 2, the main panels (insets) correspond to $\epsilon=-1(\epsilon=+1)$.

of existence is represented in all panels of Figs. 1 and 2 by the darker shaded area, being obtained by extrapolating to the continuum the results from a set of around 1000 numerical solutions.

Figure 1 (top panel) exhibits an overview of the domain of existence in an $M / \lambda$ vs $j$ plot. Consider first the limits of the domain of existence, which in fact appear in all panels of the subsequent figures. For $\epsilon=-1$, the domain is bounded by two sets of solutions: (i) the "existence line," which corresponds to the bifurcation edge from the Kerr family (see the solid blue line in Figs. 1 and 2), and (ii) the set of "critical solutions" (dotted red lines in Figs. 1 and 2) [53]. A third boundary exists when $\epsilon=+1$, the "static configurations" [41,54] (dashed-dotted black lines in the insets of Figs. 1 and 2).

The existence line is universal for any coupling function allowing for scalarization. In principle, this particular set of solutions can be found by solving the scalar field equation (as a test field) on the Kerr background. In our approach, however, the existence line is found as the limiting configuration wherein $\phi \rightarrow 0$, when varying $r_{H}, \Omega_{H}$ for 

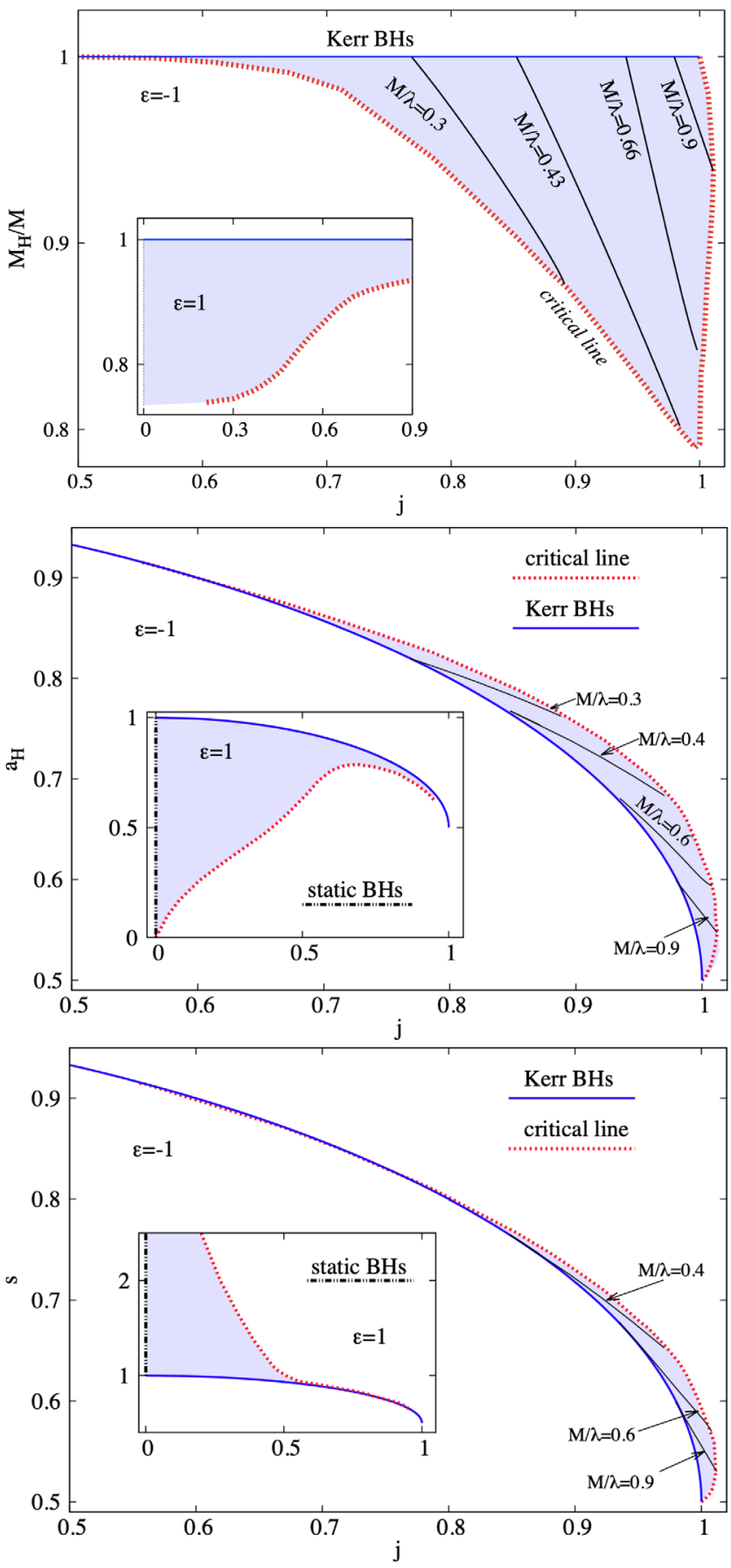

FIG. 2. Horizon mass over ADM mass $M_{H} / M$ ratio (top panel), reduced horizon area $a_{H}$ (middle panel), and reduced entropy $s$ (bottom panel) as functions of the dimensionless spin $j$.

fixed $\lambda$. Some quantitative details on the existence line are given in the Supplemental Material, Sec. IV.

The set of critical solutions is model dependent. The numerical process fails to converge as this set of configurations is approached. Typically, neither a singular behavior nor a deterioration of the numerical accuracy in the vicinity of this set was observed. The existence of such critical solutions in fairly commonplace in sGB models, both for spherical $[27,29,30]$ and rotating $[55,56]$ hairy BHs. An explanation can be traced back to the fact that the radicand of a square root in the horizon expansion of the scalar field vanishes as the critical set is approached (see e.g., Appendix A in [56] or Sec. 5.1 in [31]. As such, a consistent near horizon expansion of the solution ceases to exist, indicating that a solution that is regular there does not exist.

From Fig. 1 (top panel), we see that $\epsilon=-1$ solutions exist for a range of values of the dimensionless spin $0.5<j \lesssim 1$. Concerning the lower limit, the minimum $j$ value retrieved along the existence line with our procedure is $j \simeq 0.55$. This is compatible with the fact that the spininduced scalarization instability of Kerr can only exist for $j>0.5$ and also with the results in $[15,44,45]$. Concerning the upper limit, within the dataset collected, the maximal value of $j$ for the scalarized BHs slightly exceeds unity: $j_{\max } \sim 1.01$. This means that scalarized BHs in this model exhibit small violations of the Kerr bound. This $j$ range contrasts with the $\epsilon=+1$ case (inset), which extends down to $j=0$. As a similar trend for both $\epsilon= \pm 1$, for a given $\lambda$, the maximal allowed scalarized $\mathrm{BH}$ mass increases with $j$ (for $\epsilon=+1$ this holds for sufficiently high angular momentum). This assertion relies on the shape of the existence line, and it is thus universal for any coupling function $f(\phi)$ allowing for scalarization.

Now let us examine some of the physical properties of the solution. First, how much scalar "hair" do the scalarized solutions posses? Several quantities can be used to address this question. In Fig. 1 (bottom panel), the scalar charge (in units of $\lambda$ ) is represented against $j$. In the $\epsilon=+1$, this charge is maximized for static $j=0$ solutions. For the $\epsilon=-1$ case, it is maximized (within numerical accuracy) for $j \sim j_{\max }$, corresponding to $Q_{s} / \lambda \sim 0.038$. Comparing it to Fig. 1 (top panel), we conclude that the maximal $Q_{s}$ occurs for $M / \lambda \simeq 0.9$.

A comparison between Fig. 1 (bottom panel) and Fig. 2 (top panel) also reveals that $Q_{s}$ is no faithful measure of the fraction of the mass stored in the $\mathrm{BH}$ (and hence the fraction stored in the scalar field), as these two quantities are not extremized for the same $M / \lambda$ [57]. In this respect, Fig. 2 (top panel) shows that a significant part of the total mass is stored outside the horizon. For $\epsilon=+1$, this fraction obeys $M_{H} / M \gtrsim 0.735$, whereas for $\epsilon=-1$, $M_{H} / M \gtrsim 0.79$. This suggests significant differences in some phenomenological properties, e.g., geodesic motion and $\mathrm{BH}$ shadows, may exist with respect to comparable Kerr BHs. These difference, moreover, should be enhanced for larger $j$ up to near the maximal $j$.

An important distinction between the $\epsilon= \pm 1$ models concerns the horizon area. Figure 2 (middle panel) shows that, for the same $j, a_{H}$ is maximized (minimized) by the Kerr solution for $\epsilon=+1(\epsilon=-1)$. In this sense, spininduced scalarized BHs are larger than Kerr, whereas they are smaller in the gravitoelectric $(j \leq 0.5)$ led scalarization. 
Yet, in both cases, they are entropically favored over Kerr [see Fig. 2 (bottom panel)]. This is partly explained by the fact that the correction to the GR BH entropy depends on the sign of $f(\phi)$, cf. Eq. (8). We remark, however, that the entropic preference for the same $M, J$ in axial symmetry may be less significant for the dynamical preference than in spherical symmetry, as gravitational radiation can be emitted during the process of scalarization for the former but not the latter.

Conclusions. - We have solved the full field equations to generate solutions that describe stationary, rotating BHs in an illustrative model [cf. Eq. (3)] that exhibits the spininduced tachyonic instability found in Ref. [15]. Our results clearly demonstrate that slowly spinning stationary BHs in this model are described by the Kerr solution, whereas rapidly spinning ones exhibit scalar hair. The transition between the two classes of solutions takes place right on the threshold of the tachyonic instability found in Ref. [15]. Hence, the hairy solutions are expected to be end states of spin-induced BH scalarization.

Spin-induced scalarization raises the exciting possibility that astrophysical BHs will defy the Kerr hypothesis only for large spins, which merits further investigation. We have already established that the scalarized $\mathrm{BH}$ solutions are entropically preferred in the regime of the tachyonic instability, but it would be interesting to study their stability properties. It would also be important to follow dynamically the development of the tachyonic instability found in Ref. [15], track the formation of scalar hair, and verify explicitly that the solutions found here are the end points of this instability. This has been achieved in simpler BH scalarization scenarios [36], but it is particularly challenging when one has a coupling with the Gauss-Bonnet invariant, although significant progress has recently been made in modeling nonlinear time-domain evolutions in these theories [58-67]. Finally, the astrophysical phenomenology and implications of the scalarized BHs reported herein is missing, and our results hold the promise of non-negligible deviations from the Kerr phenomenology.

We thank $H$. Witek for discussions. C. H. and E. R. acknowledge support by the Center for Research and Development in Mathematics and Applications (CIDMA) through the Portuguese Foundation for Science and Technology (FCTFundação para a Ciência e a Tecnologia), references UIDB/04106/2020 and UIDP/04106/2020, and by national funds (OE), through FCT, I.P., in the scope of the framework contract foreseen in the numbers 4,5 , and 6 of the article 23 of the Decree-Law 57/2016 of August 29, changed by Law 57/2017 of July 19. We acknowledge support from Fundação para a Ciência e a Tecnologia the projects PTDC/FIS-OUT/28407/2017 and CERN/FISPAR/0027/2019. This work has further been supported by the European Union Horizon 2020 research and innovation (RISE) program H2020-MSCA-RISE-2017 Grant
No. FunFiCO-777740. H. O. S. and N. Y. are supported by NASA Grant Nos. NNX16AB98G, 80NSSC17M0041, and 80NSSC18K1352 and NSF Grant No. 1759615. T.P. S. acknowledges partial support from the STFC Consolidated Grant No. ST/P000703/1. We also acknowledge networking support by the COST Action GWverse Grant No. CA16104.

Note added.-Recently, we became aware of a manuscript which independently derives similar results for a different coupling function [68].

[1] B. P. Abbott et al. (LIGO Scientific and Virgo Collaborations), Phys. Rev. Lett. 116, 061102 (2016).

[2] B. P. Abbott et al. (LIGO Scientific and Virgo Collaborations), Astrophys. J. 882, L24 (2019).

[3] K. Akiyama et al. (Event Horizon Telescope Collaboration), Astrophys. J. 875, L1 (2019).

[4] R. P. Kerr, Phys. Rev. Lett. 11, 237 (1963).

[5] R. Brito, V. Cardoso, and P. Pani, Lect. Notes Phys. 906, 1 (2015).

[6] A. Arvanitaki and S. Dubovsky, Phys. Rev. D 83, 044026 (2011).

[7] C. A. R. Herdeiro and E. Radu, Phys. Rev. Lett. 112, 221101 (2014).

[8] C. Herdeiro, E. Radu, and H. Rúnarsson, Classical Quantum Gravity 33, 154001 (2016).

[9] W. E. East and F. Pretorius, Phys. Rev. Lett. 119, 041101 (2017).

[10] S. R. Dolan, Phys. Rev. D 87, 124026 (2013).

[11] H. Witek, V. Cardoso, A. Ishibashi, and U. Sperhake, Phys. Rev. D 87, 043513 (2013).

[12] S. R. Dolan, Phys. Rev. D 98, 104006 (2018).

[13] H. O. Silva, J. Sakstein, L. Gualtieri, T. P. Sotiriou, and E. Berti, Phys. Rev. Lett. 120, 131104 (2018).

[14] D. D. Doneva and S. S. Yazadjiev, Phys. Rev. Lett. 120, 131103 (2018).

[15] A. Dima, E. Barausse, N. Franchini, and T. P. Sotiriou, Phys. Rev. Lett. 125, 231101 (2020).

[16] T. Damour and G. Esposito-Farèse, Phys. Rev. Lett. 70, 2220 (1993).

[17] T. Damour and G. Esposito-Farèse, Phys. Rev. D 54, 1474 (1996).

[18] P. C. Freire, N. Wex, G. Esposito-Farèse, J. P. Verbiest, M. Bailes, B. A. Jacoby, M. Kramer, I. H. Stairs, J. Antoniadis, and G. H. Janssen, Mon. Not. R. Astron. Soc. 423, 3328 (2012).

[19] L. Shao, N. Sennett, A. Buonanno, M. Kramer, and N. Wex, Phys. Rev. X 7, 041025 (2017).

[20] D. Anderson, P. Freire, and N. Yunes, Classical Quantum Gravity 36, 225009 (2019).

[21] F. M. Ramazanoğlu and F. Pretorius, Phys. Rev. D 93, 064005 (2016).

[22] Matter in the vicinity of BHs could lead to scalarization in principle $[23,24]$, but it is not clear if astrophysical environments provide sufficiently high matter densities for the effect to take place. 
[23] V. Cardoso, I. P. Carucci, P. Pani, and T. P. Sotiriou, Phys. Rev. Lett. 111, 111101 (2013).

[24] V. Cardoso, I. P. Carucci, P. Pani, and T. P. Sotiriou, Phys. Rev. D 88, 044056 (2013).

[25] S. W. Hawking, Commun. Math. Phys. 25, 167 (1972).

[26] T. P. Sotiriou and V. Faraoni, Phys. Rev. Lett. 108, 081103 (2012).

[27] T. P. Sotiriou and S.-Y. Zhou, Phys. Rev. D 90, 124063 (2014).

[28] Prime denotes derivative with respect to the argument.

[29] P. Kanti, N. E. Mavromatos, J. Rizos, K. Tamvakis, and E. Winstanley, Phys. Rev. D 54, 5049 (1996).

[30] T. P. Sotiriou and S.-Y. Zhou, Phys. Rev. Lett. 112, 251102 (2014).

[31] J. F. Delgado, C. A. Herdeiro, and E. Radu, J. High Energy Phys. 04 (2020) 180.

[32] A famous example, in a different context, is the Breitenlohner-Freedman bound in AdS spacetimes [33].

[33] P. Breitenlohner and D. Z. Freedman, Ann. Phys. (N.Y.) 144, 249 (1982).

[34] N. Andreou, N. Franchini, G. Ventagli, and T. P. Sotiriou, Phys. Rev. D 99, 124022 (2019).

[35] G. Ventagli, A. Lehébel, and T. P. Sotiriou, Phys. Rev. D 102, 024050 (2020).

[36] C. A. R. Herdeiro, E. Radu, N. Sanchis-Gual, and J. A. Font, Phys. Rev. Lett. 121, 101102 (2018).

[37] P. G. S. Fernandes, C. A. R. Herdeiro, A. M. Pombo, E. Radu, and N. Sanchis-Gual, Phys. Rev. D 100, 084045 (2019).

[38] J. L. Blázquez-Salcedo, D. D. Doneva, J. Kunz, and S. S. Yazadjiev, Phys. Rev. D 98, 084011 (2018).

[39] H. O. Silva, C. F. B. Macedo, T. P. Sotiriou, L. Gualtieri, J. Sakstein, and E. Berti, Phys. Rev. D 99, 064011 (2019).

[40] C. F. B. Macedo, J. Sakstein, E. Berti, L. Gualtieri, H. O. Silva, and T. P. Sotiriou, Phys. Rev. D 99, 104041 (2019).

[41] P. V. P. Cunha, C. A. R. Herdeiro, and E. Radu, Phys. Rev. Lett. 123, 011101 (2019).

[42] The constant term is introduced for convenience and does not contribute to the field equations, as $\mathcal{G}$ is a total divergence. For small values of $\phi$, this theory is encompassed under the effective theory formulated in [40].

[43] C. Cherubini, D. Bini, S. Capozziello, and R. Ruffini, Int. J. Mod. Phys. D 11, 827 (2002).

[44] S. Hod, Phys. Rev. D 102, 084060 (2020).

[45] D. D. Doneva, L. G. Collodel, C. J. Krüger, and S. S. Yazadjiev, Phys. Rev. D 102, 104027 (2020).
[46] Only nonextremal solutions can be studied within the metric ansatz of Eq. (5).

[47] See Supplemental Material, which includes Ref. [48], at http://link.aps.org/supplemental/10.1103/PhysRevLett.126 .011103 for further details on the theory's field equations, numerical methods, and the physical properties of some of the solutions.

[48] W. Schonauer and R. Weiss, J. Comput. Appl. Math. 27, 279 (1989).

[49] S. R. Coleman, J. Preskill, and F. Wilczek, Nucl. Phys. B378, 175 (1992).

[50] C. A. Herdeiro and E. Radu, Int. J. Mod. Phys. D 24, 1542014 (2015).

[51] J. Kunz, I. Perapechka, and Y. Shnir, Phys. Rev. D 100, 064032 (2019).

[52] R. M. Wald, Phys. Rev. D 48, R3427 (1993).

[53] The critical solutions interpolating between the scalarized $\mathrm{BH}$ with maximal $j$ and extremal Kerr BHs have a small Hawking temperature, which suggests the possible existence of a (small) set of extremal BHs. However, this issue has proved to be numerically difficult to explore.

[54] L. G. Collodel, B. Kleihaus, J. Kunz, and E. Berti, Classical Quantum Gravity 37, 075018 (2020).

[55] B. Kleihaus, J. Kunz, and E. Radu, Phys. Rev. Lett. 106, 151104 (2011).

[56] B. Kleihaus, J. Kunz, S. Mojica, and E. Radu, Phys. Rev. D 93, 044047 (2016).

[57] The horizon mass $M_{H}$ is computed as a Komar integral.

[58] R. Benkel, T. P. Sotiriou, and H. Witek, Phys. Rev. D 94, 121503(R) (2016).

[59] R. Benkel, T. P. Sotiriou, and H. Witek, Classical Quantum Gravity 34, 064001 (2017).

[60] H. Witek, L. Gualtieri, P. Pani, and T. P. Sotiriou, Phys. Rev. D 99, 064035 (2019).

[61] M. Okounkova, Phys. Rev. D 100, 124054 (2019).

[62] M. Okounkova, Phys. Rev. D 102, 084046 (2020).

[63] J. L. Ripley and F. Pretorius, Phys. Rev. D 101, 044015 (2020).

[64] J. L. Ripley and F. Pretorius, Classical Quantum Gravity 36, 134001 (2019).

[65] J. L. Ripley and F. Pretorius, Classical Quantum Gravity 37, 155003 (2020).

[66] H. Witek, L. Gualtieri, and P. Pani, Phys. Rev. D 101, 124055 (2020).

[67] F.-L. Julié and E. Berti, Phys. Rev. D 101, 124045 (2020).

[68] E. Berti, L. G. Collodel, B. Kleihaus, and J. Kunz, following Letter, Phys. Rev. Lett. 126, 011104 (2021). 